\title{
Exotic gapless spectrum induced by frustration in quantum antiferromagnets
}

\author{
Federico Becca, ${ }^{1}$ Luca Capriotti, ${ }^{2}$ Alberto Parola, ${ }^{3}$ and Sandro Sorella ${ }^{1}$ \\ ${ }^{1}$ CNR-INFM-Democritos National Simulation Centre and International School for Advanced Studies (SISSA), \\ Via Beirut 2-4, I-34014 Trieste, Italy \\ ${ }^{2}$ Global Modelling and Analytics Group, Investment Banking Division, Credit Suisse Group, \\ One Cabot Square, London, E14 4QJ, United Kingdom \\ ${ }^{3}$ Dipartimento di Fisica e Matematica, Università dell'Insubria, Via Valleggio 11, I-22100 Como, Italy
}

(Received 6 June 2007; published 2 August 2007)

\begin{abstract}
We show strong numerical evidence in favor of an unexpected virtually gapless spectrum, with edge states localized at the boundaries, in frustrated spin-1/2 antiferromagnetic ladders with an odd number of legs. These features can be accurately reproduced by using a projected BCS wave function with a nontrivial pairing that mixes even and odd reflection symmetries. This approach gives the correct classification of the excitations and provides a simple and very appealing picture of an unconventional spin-liquid phase stabilized by frustration.
\end{abstract}

In the last few decades there has been an increasing theoretical and experimental effort to clarify the nature of the disordered phases stabilized by competing interactions in magnetic materials. ${ }^{1}$ A very exciting scenario appears when such disordered spin liquids are gapless with fractional excitations, generalizing the critical phase of one-dimensional systems to higher dimensions. ${ }^{2}$ Recent realizations of frustrated antiferromagnets on quasi-two-dimensional lattices, like $\mathrm{NiGa}_{2} \mathrm{~S}_{4}$ or $\kappa$ - $(\mathrm{ET})_{2} \mathrm{Cu}_{2}(\mathrm{CN})_{3}{ }^{3,4}$ give promising evidence in this direction. In particular, in organic materials, NMR shows a power-law behavior of $1 / T_{1}$ at very low temperatures without any signal of magnetic order. ${ }^{5}$ Interestingly, also in $\mathrm{Cs}_{2} \mathrm{CuCl}_{4}$, neutron scattering measurements show the existence of a continuum of excited states, compatible with pairs of spinon excitations. ${ }^{6}$

From a theoretical point of view, a promising strategy is to consider quasi-one-dimensional systems, where several very reliable techniques are available, e.g., bosonization or density-matrix renormalization group (DMRG), in order to extract important insight into the more relevant twodimensional (2D) case. In this Rapid Communication, we take this point of view and study the spin-1/2 $J_{1}-J_{2}$ Heisenberg model on ladders with an odd number of legs,

$$
\mathcal{H}=J_{1} \sum_{\left\langle R, R^{\prime}\right\rangle} \mathbf{S}_{R} \cdot \mathbf{S}_{R^{\prime}}+J_{2} \sum_{\left\langle\left\langle R, R^{\prime}\right\rangle\right\rangle} \mathbf{S}_{R} \cdot \mathbf{S}_{R^{\prime}}
$$

where $\mathbf{S}_{R}$ is the spin operator on site $R=(x, y)$, and the sum is restricted to first $\left(J_{1}\right)$, second $\left(J_{2}\right)$ nearest neighbors. We consider systems with $N=L \times n$ sites, where $n$ is the odd number of legs and $L$ is the number of rungs, with open boundary conditions along the rungs.

The physical properties of odd-leg ladders are expected to be similar to the ones of the $2 \mathrm{D}$ case. Indeed, although for any finite number of legs $n$ there are no magnetically ordered phases, in the weakly frustrated regime, i.e., $J_{2} / J_{1} \ll 1$ or $J_{1} / J_{2} \ll 1$, the excitation spectrum has a gapless branch, analogously to the $2 \mathrm{D}$ case. Such a system, for small $n$, has been recently considered by different groups with contradicting results. ${ }^{7-9}$ In particular, in Ref. 8 it has been argued that, for any number of legs, a relevant operator that breaks the translational symmetry induces spontaneous dimerization in the intermediate region $J_{2} / J_{1} \sim 1 / 2$. On the contrary, such a dimerization has not yet been detected by numerical calculations. ${ }^{9}$ In order to clarify this issue, we consider the model of Eq. (1) with $n=3$ by using a variety of numerical techniques. Lanczos exact diagonalizations unveil a very peculiar and unexpected excitation spectrum and DMRG rules out any sizable dimerization. Remarkably, all these numerically exact results can be interpreted by means of a simple, but nevertheless extremely accurate, projected BCS state, with a nontrivial pairing function.

Let us start with the excitation spectrum obtained by Lanczos diagonalizations with periodic boundary conditions along the chains. In Fig. 1, we show the evolution of a few low-energy excitations in the relevant subspaces defined by

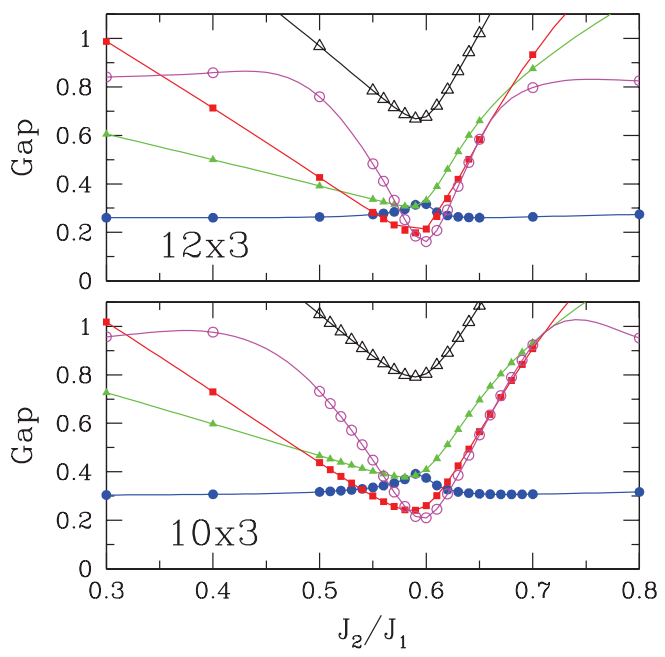

FIG. 1. (Color online) Some of the relevant low-energy gaps as a function of $J_{2} / J_{1}$ from Lanczos data. Triplet with $k_{x}=\pi, r_{x}=1$, and $r_{y}=1$ (blue circles), singlet with $k_{x}=\pi, r_{x}=-1$, and $r_{y}=1$ (green triangles), singlet with $k_{x}=0, r_{x}=1$, and $r_{y}=-1$ (red squares), singlet with $k_{x}=\pi, r_{x}=1$, and $r_{y}=-1$ (empty black triangles), and the lowest excitation in the same subspace of the ground state (empty magenta circles). All the quantum numbers are referred to the ground state and lines are guides to the eye. 


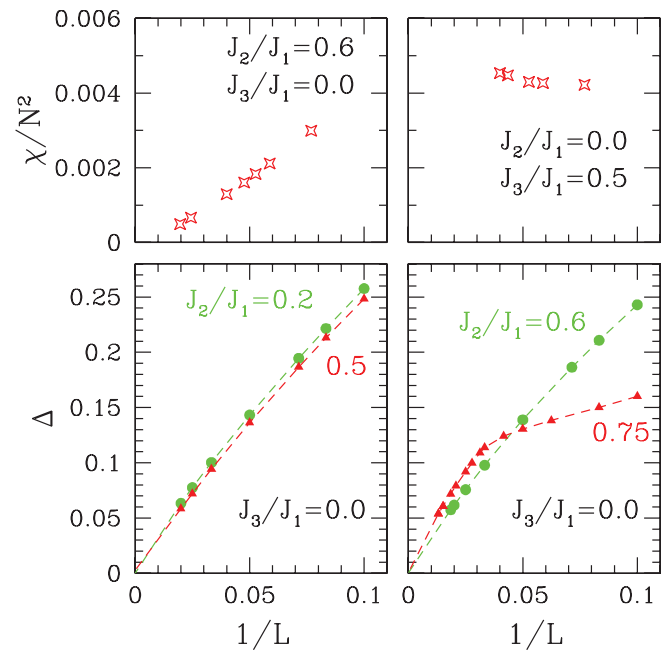

FIG. 2. (Color online) Size scaling for the dimer susceptibility $\chi$ (upper panels) and for the triplet gap $\Delta$ (lower panels) evaluated by DMRG.

the spatial symmetries of the Hamiltonian: the crystal momentum along the $x$ direction, the reflection $\mathcal{R}_{x}$ that changes $x \rightarrow-x$, the reflection $\mathcal{R}_{y}$ across the central chain, and the total spin. For both small and large $J_{2}$, as expected, all the excitations odd under $\mathcal{R}_{y}$ (i.e., $r_{y}=-1$ ) have a sizable gap. Although the quantum numbers of the ground state do not change by varying $J_{2} / J_{1}$, we have evidence in favor of an avoided crossing that is indicated by the sudden drop of the lowest energy gap in the ground-state subspace. This suggests the possible occurrence of a first-order phase transition, slightly shifted to larger values of $J_{2} / J_{1}$ (i.e., $J_{2} / J_{1} \sim 0.6$ ) with respect to the classical model. ${ }^{1}$ More interestingly, the frustrating interaction induces a dramatic effect on excitations with odd $\mathcal{R}_{y}$, and for $J_{2} / J_{1} \sim 0.55$ the lowest excitation is an $r_{y}=-1$ singlet with zero momentum $k_{x}$ (referenced to the ground state). Although Lanczos diagonalizations are limited to $12 \times 3$, we have evidence that, in the thermodynamic limit, this excitation becomes gapless in a small region around $J_{2} / J_{1} \sim 0.55$. We notice that the quantum numbers of this excitation are not the ones implied by the Affleck-Lieb-Schulz-Mattis theorem, i.e., a gapless state with momentum $k_{x}=\pi$ with respect to the ground state. ${ }^{10} \mathrm{In}$ agreement with that, we also have evidence that both a triplet state $\left(r_{x}=1\right.$ and $\left.r_{y}=1\right)$ and a singlet state $\left(r_{x}=-1\right.$ and $\left.r_{y}=1\right)$ with $k_{x}=\pi$ become gapless in the thermodynamic limit for all the values of $J_{2} / J_{1}$ considered. These results are not compatible with conventional dimerization, where the ground state is doubly degenerate with a finite gap with respect to all the other excitations.

A further confirmation of a gapless triplet excitation comes from a systematic finite-size scaling by using DMRG with open boundary conditions in both $x$ and $y$ directions. The triplet gap vanishes in the thermodynamic limit for all the values of $J_{2} / J_{1}$ considered (see Fig. 2), in agreement with previous DMRG results. ${ }^{9}$ In order to reconcile the numerical finding with the theoretical predictions of Ref. 8, we consider the influence of a third-nearest-neighbor interaction $J_{3}$. In fact, as suggested in Ref. 11, for $J_{2}=0$ this stabilizes a phase

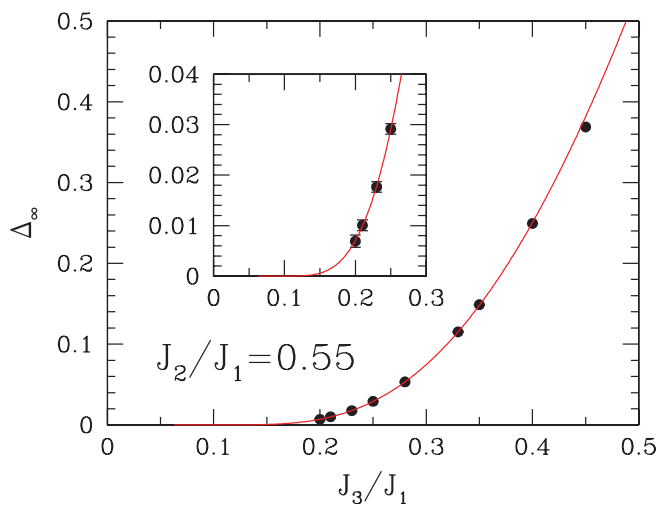

FIG. 3. (Color online) Triplet gap $\Delta_{\infty}$ as a function of $J_{3} / J_{1}$ in the thermodynamic limit, from DMRG data. The line is a fit according to the KT theory, $\Delta_{\infty}=A \exp \left[-b / \sqrt{\left(J_{3}-J_{3}^{c}\right) / J_{1}}\right]$. Inset: Detail for $J_{3} / J_{1}<0.3$.

with a sizable gap and dimerization. In this respect, we have studied the size scaling of the spin gap for $J_{2} / J_{1}=0.55$ for different values of $J_{3} / J_{1}$. As shown in Fig. 3, the thermodynamic limit of the spin gap is clearly finite for $J_{3} / J_{1} \geqslant 0.2$, and vanishes as $J_{3}$ decreases. Since the spin gap is characterized by a Kosterlitz-Thouless (KT) behavior, the precise value of the critical coupling $J_{3}^{c}$ is difficult to assess numerically. However, our results indicate that for $J_{3} / J_{1} \lesssim 0.1$ the spin gap is exceedingly small $\left(\lesssim 10^{-8} J_{1}\right)$. In addition, extensive calculations on five- and seven-leg ladders indicate that the finite-size gap at fixed $L$ decreases with the number of legs, thus suggesting the stabilization of a gapless and homogeneous phase for $J_{2} / J_{1} \simeq 0.55$ also in $2 \mathrm{D}$. This conclusion is also confirmed by a study of the dimer susceptibility calculated by adding a small perturbation that breaks the translational symmetry, $O=\delta \Sigma_{R} e^{i Q R} \mathbf{S}_{R} \cdot \mathbf{S}_{R+x}$, with $Q=(\pi, 0)$, and by computing the second derivative of the ground-state energy with respect to $\delta$ (see Fig. 2). A clear dimerization is found for $J_{3} / J_{1}=0.5$ but not for $J_{3}=0$, as in the latter case the dimer susceptibility does not display the sharp $\chi \propto N^{2}$ divergence required in a symmetry broken phase. ${ }^{12}$

In the following, we will show that a simple variational ansatz is able to explain the gross features of the anomalous low-energy spectrum. Our construction is based on a projected BCS (pBCS) wave function:

$$
|\mathrm{pBCS}\rangle=\mathcal{P}|\mathrm{BCS}\rangle,
$$

where $\mathcal{P}$ is the projector onto the subspace of single occupied sites, and $|\mathrm{BCS}\rangle$ is the ground state of

$$
\mathcal{H}_{\mathrm{BCS}}=-t \sum_{\left\langle R, R^{\prime}\right\rangle \sigma} c_{R, \sigma}^{\dagger} c_{R^{\prime}, \sigma}+\sum_{R, R^{\prime}} \Delta_{R, R^{\prime}} c_{R, \uparrow}^{\dagger} c_{R^{\prime}, \downarrow}^{\dagger}+\text { H.c. }
$$

where $c_{R, \sigma}^{\dagger}\left(c_{R, \sigma}\right)$ creates (destroys) an electron of spin $\sigma$ at site $R=(x, y), t=1$ is the nearest-neighbor hopping amplitude, and $\Delta_{R, R^{\prime}}=\Delta_{R^{\prime}, R}$ are real singlet pairings determining the symmetry of the BCS order parameter. If the pairing involves only nearest-neighbors sites along the coordinate directions, $\Delta^{x}$ and $\Delta^{y}$ (even under both reflections $\mathcal{R}_{x}$ and $\mathcal{R}_{y}$ ), the BCS eigenstates can be labeled by further quantum 
numbers, i.e., $p_{h}= \pm 1$, associated to the particle-hole transformation

$$
P_{h} c_{R, \sigma}^{\dagger} P_{h}=\operatorname{sign}(\sigma)(-1)^{x+y} c_{R,-\sigma},
$$

which commutes with both $\mathcal{H}_{\mathrm{BCS}}$ and $\mathcal{P}$, and the projected state (2) has the same spatial symmetries of the spin Hamiltonian. However, the physical states that survive after projection are only those with $p_{h}=1$ for even $S^{z}$ or $p_{h}=-1$ for odd $S^{z}$. Therefore all physical states have a given $P_{h}$ according to their $z$ component of the total spin. In the BCS Hamiltonian, we have the freedom to take either periodic boundary conditions (PBC), i.e., $c_{x, y}=c_{x+L, y}$, or antiperiodic ones (APBC), i.e., $c_{x, y}=-c_{x+L, y}$. Of course, in both cases, after projection the wave function (2) describes a spin state consistent with PBC. The lowest-energy state is obtained with PBC for $L$ $=4 m+2$ and with APBC for $L=4 m$, while the lowest excitations correspond to the Gutzwiller projection of the BCS ground states with the other choice of the boundary conditions, namely APBC (PBC) for $L=4 m+2(L=4 m)$. In this case, the ground state of Eq. (3) is degenerate because of the presence of four zero-energy Bogoliubov modes that can be identified as spinons, carrying spin $1 / 2$ and momenta $k_{x}$ $= \pm \pi / 2$. With these objects, we can construct four zeroenergy states with $p_{h}=1$ (that survive after projection): One triplet and three singlets. Indeed, two spinons can form (i) a triplet of momentum $k_{x}=\pi$ even under $\mathcal{R}_{x}$, (ii) one singlet with $k_{x}=\pi$ odd in $\mathcal{R}_{x}$, and (iii) one singlet with $k_{x}=0$ even under $\mathcal{R}_{x}$. A further singlet can be obtained by combining zero and four spinons, yielding (iv) a state with momentum $k_{x}=\pi$ and odd with respect to $\mathcal{R}_{x}$. All these states have $\mathcal{R}_{y}$ even. The two singlet states (ii) and (iv), belonging to the same symmetry subspace, do not represent distinct excitations: In fact, numerical calculations show that their overlap increases with the size of the system. Analogously, the singlet (iii) is asymptotically identical to the ground state. Therefore, out of the four distinct states, we just obtain two independent excitations: A triplet and a singlet, precisely reproducing the lowest level of the "tower of states" predicted by conformal field theory in the nonfrustrated case. ${ }^{13}$

Much more interesting is the case when the BCS Hamiltonian breaks some symmetry that is instead restored after projection. For instance, as already emphasized in $2 \mathrm{D},{ }^{14}$ we can add a next-nearest-neighbor pairing $\Delta^{x y}$, with odd reflection symmetry under $\mathcal{R}_{x}$ and $\mathcal{R}_{y}$. In this case, both reflection and particle-hole symmetry (4) do not commute with $\mathcal{H}_{\mathrm{BCS}}$ because of the simultaneous presence of $\Delta^{x}, \Delta^{y}$, and $\Delta^{x y}$. Instead, after projection onto the physical subspace with singly occupied sites, these symmetries are restored. Indeed, in this case, the BCS Hamiltonian is invariant under $\widetilde{\mathcal{R}}_{x}$ $=P_{h} \mathcal{R}_{x}$ and $\widetilde{\mathcal{R}}_{y}=P_{h} \mathcal{R}_{y}$. Therefore the ground state of $\mathcal{H}_{\mathrm{BCS}}$ has a well-defined value of $\widetilde{\mathcal{R}}_{x}$ and $\widetilde{\mathcal{R}}_{y}$ and, after projection, since $P_{h}$ is the identity in the physical Hilbert space, it has also well-defined values of the true reflection symmetries $\mathcal{R}_{x}$ and $\mathcal{R}_{y}$. Since $P_{h}$ is no longer a symmetry of $\mathcal{H}_{\mathrm{BCS}}$, the eigenstates that were previously forbidden in the physical spectrum due to their wrong value of $P_{h}$ are instead now allowed. This approach predicts to a pair of $r_{y}=-1$ excitations not present in the usual bosonization analysis: They are

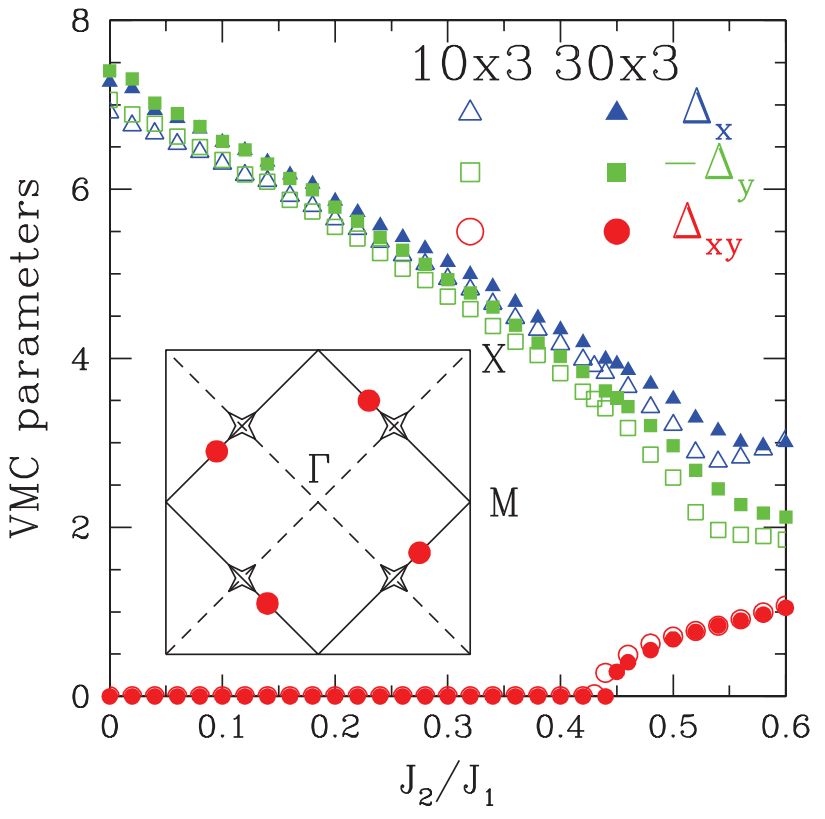

FIG. 4. (Color online) "Phase diagram" of the $J_{1}-J_{2}$ three-leg ladder found by the variational pBCS state (see text). The Brillouin zone for the 2D lattice is shown in the inset: Stars indicate 2D gapless spinon excitations for $\Delta^{x y}=0[$ at $( \pm \pi / 2, \pm \pi / 2)]$ and red dots for $\Delta^{x y} \neq 0$.

both singlets, even with respect to $\mathcal{R}_{x}$ and have different momenta: (v) $k_{x}=0$ and (vi) $k_{x}=\pi$. Remarkably, the first of these states has precisely the same quantum numbers of the lowest excitation emerging in the frustrated regime $J_{2} / J_{1} \sim 0.55$, while the second one, although higher in energy, has a sudden drop in the same region, see Fig. 1.

Further insight on the physics underlying this result comes from the structure of the BCS excitations. The key feature induced by a nonvanishing $\Delta^{x y}$ coupling is the localization of the spinon wave function $\psi_{j}$ near the edges of the ladder (here $j$ labels the number of the leg): $\psi_{2 j}=0$ and $\psi_{2 j+1}=\tau^{j}$ with $\tau=-\left(t-2 \Delta^{x y}+i \Delta^{y}\right) /\left(t+2 \Delta^{x y}+i \Delta^{y}\right)$. Only in the $\Delta^{x y} \rightarrow 0$ limit we get $\tau=-1$ and the spinon delocalizes among the chains. Instead, if $|\tau| \neq 1$, the gapless spinons become edge states. This scenario is confirmed by a DMRG study on three- and five-leg ladders with an odd number of sites per leg, so to have a free spinon trapped in the lattice.

After this detailed exam of the general properties of the pBCS wave function, we show in Fig. 4 the "phase diagram" of the three-leg ladder, obtained by variational Monte Carlo optimization. The optimal couplings $\Delta^{x}, \Delta^{y}$, and $\Delta^{x y}$ have been numerically determined. The most relevant feature is the stabilization of a nonvanishing $\Delta^{x y}$ for $J_{2} / J_{1} \geq 0.44$. The variational estimate of the transition point is likely to be somewhat inaccurate, e.g., for one chain, spontaneous dimerization is found for $J_{2} / J_{1} \gtrsim 0.15$ instead of $J_{2} / J_{1} \sim 0.24$. Nevertheless we believe that the global trends emerging from this phase diagram are representative of the real behavior of the model. Furthermore, we checked that dimer correlations do not display any clear tendency towards ordering. Note that, whenever the spinon spectrum is gapped (e.g., when 
TABLE I. The low-energy states predicted by the pBCS approach compared with the exact diagonalization data for $J_{2} / J_{1}$ $=0.55$ on the $10 \times 3$ ladder. The variational parameters are $\Delta^{x}$ $=1.438, \Delta^{y}=-0.987$, and $\Delta^{x y}=0.457$.

\begin{tabular}{lccccccc}
\hline \hline Boundary & $k_{x}$ & $r_{x}$ & $r_{y}$ & Spin & $E_{v m c}$ & $E_{0}$ & Overlap \\
\hline PBC & 0 & 1 & 1 & 0 & -14.1281 & -14.2042 & 0.969 \\
APBC & 0 & 1 & -1 & 0 & -13.7997 & -13.9038 & 0.972 \\
APBC & $\pi$ & 1 & 1 & 1 & -13.7532 & -13.8689 & 0.923 \\
APBC & $\pi$ & -1 & 1 & 0 & -13.6404 & -13.8023 & 0.932 \\
APBC & $\pi$ & 1 & -1 & 0 & -13.0647 & -13.3265 & 0.882 \\
\hline \hline
\end{tabular}

$\mathrm{PBC}$ are taken along the rungs), the same $\mathrm{pBCS}$ wave function describes a dimerized state for finite $n .^{15}$

Finally, by considering the set of variational parameters obtained by Monte Carlo optimization of the ground-state wave function, we evaluated the elementary excitations on the $10 \times 3$ ladder by a change in the boundary conditions along the chain, without further optimization. In this way, besides the ground state, we obtained an explicit form of the excited states (i), (ii), (v), and (vi) discussed previously. The variational energies and the overlaps with the exact lowest eigenstates are shown in Table I for $J_{2} / J_{1}=0.55$. The impressive accuracy of the pBCS wave function for the full set of low-lying states provides clear evidence in favor of the variational picture.
In conclusion, our results point toward the existence of gapless phases in the three-leg spin-1/2 $J_{1}-J_{2}$ Heisenberg antiferromagnet for all values of the next-nearest-neighbor frustrating interaction. An unconventional gapless phase with an excitation spectrum characterized by the presence of lowenergy edge states is stabilized upon frustration. Although we cannot exclude the possibility of an exponentially small dimerization (e.g., with a spin gap for three legs of order $10^{-8} J_{1}$ ), we believe that the qualitative features that we found faithfully characterize the physics of the system. This homogeneous phase is accurately described by a projected BCS wave function representing an algebraic spin liquid. This scenario is even more plausible in $2 \mathrm{D}$, especially considering that the exponentially small tail of the gap implied by the KT behavior should disappear and that the same type of wave function remains accurate when increasing the (odd) number $n$ of legs. The strongly frustrated region in $2 \mathrm{D}$ is then a gapless state with incommensurate spinon excitations, that naturally result from the stabilization of a finite $\Delta^{x y},{ }^{14}$ see Fig. 4. Such an exotic spin spectrum can be experimentally detected in $2 \mathrm{D}$ frustrated antiferromagnets, like $\mathrm{Li}_{2} \mathrm{VOSiO}_{4}$ and $\mathrm{VOMoO}_{4}$ under pressure. ${ }^{16,17}$

We thank M. Fabrizio, A.A. Nersesyan, and O.A. Starykh for interesting discussions. This work has been partially supported by CNR-INFM and MIUR (COFIN 2004 and 2005).
${ }^{1}$ G. Misguich and C. Lhuillier, in Frustrated Spin Systems, edited by H. T. Diep (World-Scientific, Singapore, 2005).

${ }^{2}$ X.-G. Wen, Phys. Rev. B 44, 2664 (1991).

${ }^{3}$ S. Nakatsuji, Y. Nambu, H. Tonomura, O. Sakai, S. Jonas, C. Broholm, H. Tsunetsugu, Y. Qiu, and Y. Maeno, Science 309, 1697 (2005).

${ }^{4}$ Y. Shimizu, K. Miyagawa, K. Kanoda, M. Maesato, and G. Saito, Phys. Rev. Lett. 91, 107001 (2003).

${ }^{5}$ Y. Shimizu, K. Miyagawa, K. Kanoda, M. Maesato, and G. Saito, Phys. Rev. B 73, 140407(R) (2006).

${ }^{6}$ R. Coldea, D. A. Tennant, A. M. Tsvelik, and Z. Tylczynski, Phys. Rev. Lett. 86, 1335 (2001).

${ }^{7}$ P. Azaria, P. Lecheminant, and A. A. Nersesyan, Phys. Rev. B 58, R8881 (1998).

${ }^{8}$ O. A. Starykh and L. Balents, Phys. Rev. Lett. 93, 127202 (2004).

${ }^{9}$ X. Wang, N. Zhu, and C. Chen, Phys. Rev. B 66, 172405 (2002).
${ }^{10}$ E. H. Lieb, T. D. Schultz, and D. C. Mattis, Ann. Phys. (N.Y.) 16, 407 (1961); I. Affleck, Phys. Rev. B 37, 5186 (1988).

${ }^{11}$ L. Capriotti, D. J. Scalapino, and S. R. White, Phys. Rev. Lett. 93, 177004 (2004)

${ }^{12}$ L. Capriotti, Int. J. Mod. Phys. B 15, 1799 (2001).

${ }^{13}$ I. Affleck, D. Gepner, H. J. Schulz, and T. Ziman, J. Phys. A 22, 511 (1989).

${ }^{14}$ L. Capriotti, F. Becca, A. Parola, and S. Sorella, Phys. Rev. Lett. 87, 097201 (2001).

${ }^{15}$ S. Sorella, L. Capriotti, F. Becca, and A. Parola, Phys. Rev. Lett. 91, 257005 (2003).

${ }^{16}$ R. Melzi, P. Carretta, A. Lascialfari, M. Mambrini, M. Troyer, P. Millet, and F. Mila, Phys. Rev. Lett. 85, 1318 (2000).

${ }^{17}$ P. Carretta, N. Papinutto, C. B. Azzoni, M. C. Mozzati, E. Pavarini, S. Gonthier, and P. Millet, Phys. Rev. B 66, 094420 (2002). 